\title{
Diagnosis and treatment of metastatic alveolar echinococcosis in the brain of a Tibetan patient : A case report
}

\section{Chaonan Du}

Qinghai University

\section{Qiang Zhang}

Qinghai Provincial People's Hospital

Mingfei Yang ( $\square$ iloveyoucmu@163.com )

Qinghai Provincial People's Hospital

\section{Xiumin Han}

Qinghai Provincial People's Hospital

\section{Yanqiu Sun}

Qinghai Provincial People's Hospital

\section{Zhanying Ju}

Qinghai Provincial People's Hospital

\section{Xiaoxing Wei}

Qinghai University

\section{Research}

Keywords: Brain alveolar echinococcosis, Surgical treatment, Case analysis, Molecular identification

Posted Date: January 3rd, 2020

DOl: https://doi.org/10.21203/rs.2.19913/v1

License: (9) This work is licensed under a Creative Commons Attribution 4.0 International License. Read Full License 


\section{Abstract}

Background: A case of primary alveolar echinococcosis (AE) in the liver with kidney and brain metastases was treated at our hospital. The analysis of this case might provide suggestions for the diagnosis and treatment of other cases of $\mathrm{AE}$ in the brain.

Methods : To determine the disease status of $A E$ in the liver and brain in this patient, computed tomography (CT) of the abdomen and head magnetic resonance imaging (MRI) were performed before surgery. Additionally, the serum of the patient was analyzed using an enzyme linked immunosorbent assay (ELISA) for antibodies against echinococcus. The left pterional approach was selected for head surgery. Along the edema zone around the lesion, complete excision was performed encompassing an area of about $5 \mathrm{~mm}$ surrounding the lesion. After surgery, pathological and immunohistochemical examinations of the lesion were performed, and deoxyribonucleic acid (DNA) was extracted for molecular identification. This patient was followed up regularly post-discharge to evaluate the outcome.

Results: Anti-echinococcus antibodies were strongly positive in the patient's serum, and the eosinophil ratio was $20 \%$. The head MRI showed a single lesion in the left temporal lobe. Multiple nodular and small cystic long T 1 short T 2 signals were seen in the lesions. An enhanced scan indicated multiple ringenhancements, the boundary was unclear, and the size of lesion was $3.2 \mathrm{~mm} \times 1.8 \mathrm{~mm}$. Neurological symptoms improved after the surgery. The result of histopathological examination was consistent with the characteristics of AE. Immunohistochemistry showed that the peripheral glial cells were positive for glial fibrillary acidic protein (GFAP) and the Ki-67 index was $<3 \%$. DNA analysis identified the parasite as the Asian strain of Echinococcus multilocularis, and the sequence identity with the reference strain was practically $100.00 \%$. Conclusion: Surgery is still the first choice of treatment for single, superficial $A E$ lesions, and those located in nonfunctional areas of the brain. It is suggested that early radical treatment and standard drug treatment of primary hepatic AE are important steps to avoid secondary encephalic AE. Patients need to take albendazole for a long time after the operation, and should be followed-up regularly.

\section{Background}

Alveolar echinococcosis (AE) caused by echinococcus multilocularis is a chronic and potentially lethal parasitic disease. In the 1990s, studies of the distribution of human parasites showed that the QinghaiTibet Plateau (including Sichuan, Qinghai and parts of Gansu), Xinjiang and Ningxia Autonomous Region were highly affected endemic areas of echinococcosis ${ }^{[1]}$. Recent global data showed that there are about 18000 new $A E$ cases per year, $91 \%$ of which were diagnosed in China ${ }^{[2]}$. Because AE usually originates in the liver, it often involves the lungs, brain, bone and other organs, while central nervous system involvement accounts for only $1-3 \%$ of the cases reported in the literature ${ }^{[3]}$. For example, Altinors et al. ${ }^{[4]}$ showed that the number of cases involving the nervous system accounted for only $2-3 \%$. AE can lead to high-level disability and even death if it cannot be diagnosed and treated correctly in the early stage ${ }^{[5-}$ 
6]. Therefore, early radical treatment of liver $A E$ is particularly important. Recently, a case of primary liver AE with kidney and brain metastasis was treated in our hospital. The diagnosis, treatment and outcome of this disease were analyzed as follows.

\section{Methods}

\section{Clinical data}

The patient is a 52 year-old male Tibetan herdsman, born and living in Gander County, Golog Tibetan Autonomous Prefecture, Qinghai Province of China, which is one of those most highly endemic areas of $A E^{[7-8]}$. He was admitted to the hospital on January 17, 2019 due to "headache for 1 month, aggravating with convulsions for 1 day ". The patient had no obvious causes of the headache, which had the left temporal side as the main area, and did not receive special treatment. The symptoms were recurrent. One day before admission, the symptoms of headache were more severe than before and accompanied by epilepsy. In July 2009, the patient was diagnosed as having liver AE and received liver hydatid cystectomy and cholecystectomy. The diagnosis based on pathological examination was liver $\mathrm{AE}$, and the patient received no medication such as oral albendazole after the operation. In September 2013, the patient suffered from pain in the right lumbar and costal region. He underwent abdominal CT examination in the hospital in our province. As a result, the patient was diagnosed as having right renal echinococcosis and received a right nephrectomy. After 2 months of intermittent oral administration of albendazole, the patient stopped taking the medicine on his own.

\section{Examination}

\section{Image analysis}

The patient was examined by abdominal CT and brain MRI to confirm the status of intracranial and abdominal echinococcosis according to the diagnosis and expert consensus of echinococcosis in the central nervous system in China ${ }^{[9]}$. According to the PNM typing of liver alveolar echinococcosis proposed by the $\mathrm{WHO}^{[10]}$ and the EMUC-US typing based on ultrasonography ${ }^{[11]}$, the patient was classified as having alveolar echinococcosis of the liver. The CT was performed using a Revolution CT scanner with 64 detector rows (General Electric Company GE, USA) and the MRI analysis used a MAGNETOM Skyra 3.0T Magnetic Resonance Imaging System (Siemens Healthineers USA). The patient previously had liver and kidney echinococcosis. In order to clarify the systemic organ metastases, a $\mathrm{PET} / \mathrm{CT}$ examination was suggested, but the patient refused the examination.

\section{Serological Detection}

ELISA was used to investigate the presence of parasite-specific antibodies in the serum. Serum samples were collected from 2-5 $\mathrm{ml}$ aliquots of venous blood and tested for anti-echinococcus antibodies using an echinococcosis IgG antibody detection kit (Zhuhai Haitai Biopharmaceutical Co., Ltd., batch number 
20181102). An XT-1800i hematology analyzer (Sysmex Company, Japan) was used for routine blood tests.

\section{Pathological and Immunological Examination}

The lesion samples were fixed with $4 \%$ formaldehyde, followed by routine dehydration, paraffin embedding, slicing into $4 \mathrm{~mm}$ thick sections, hematoxylin-eosin staining (HE) and GFAP staining for microscopic observation.

\section{Genetic Testing}

The tissue DNA extraction kit (Tiangen Biochemical Technology (Beijing) Co., Ltd) was used to extracted genomic DNA. The specific operation steps were conducted in accordance with the kit's instructions. The extracted genomic DNA was used as template and the specific primers COX1F (5'-

TTGAATTTGCCACGTTTGAAT GC-3') and COX1R (5'- GAACCTAACGACATAACATAATGA -3') were used for polymerase chain reaction (PCR) amplification.

The reaction system $(50.0 \mu \mathrm{L})$ contained PCR SuperMix $(25.0 \mu \mathrm{L})$, upstream and downstream primers $(10.0 \mu \mathrm{M} ; 2.0 \mu \mathrm{L})$, template DNA $(2.0 \mu \mathrm{L})$ and water $(19.0 \mu \mathrm{L})$. The reaction conditions were as follows: initial denaturation at $94^{\circ} \mathrm{C}$ for $5 \mathrm{~min}$, followed by 35 cycles of denaturation at $94^{\circ} \mathrm{C}$ for $30 \mathrm{~s}$, annealing at $55^{\circ} \mathrm{C}$ for $30 \mathrm{~s}$, extension at $72^{\circ} \mathrm{C}$ for $1 \mathrm{~min}$, and a final extension at $72^{\circ} \mathrm{C}$ for 10 minutes. After amplification, the PCR product was separated by electrophoresis in a 1.5\% agarose gel and photographed. After sequencing and identification, a phylogenetic tree (Kimura 2 parameter model, 2000 repeated bootstrap tests) was constructed using the Neighbor Joining method in Molecular Evolutionary Genetics Analysis (MEGA) 5.0 software.

\section{Differential diagnosis}

Lumbar puncture was performed and $5 \mathrm{ml}$ of cerebrospinal fluid was collected for examination to exclude intracranial infection and tuberculosis. Cerebral angiography (CTA) and chest CT were performed to exclude cerebrovascular diseases and pulmonary metastasis.

\section{Surgery and drug therapy}

After admission, albendazole tablets were administered, $60 \mathrm{mg}$ once, twice a day. According to the diagnosis and expert consensus on echinococcosis in central nervous system in China ${ }^{[9]}$, the authors excluded the contraindications to operation. Microsurgery was used and the left pterional approach was selected, with routine craniotomy, suspension of the dura mater, "Y" type incision of the dura mater and positioning of the $\$ 88$ microscope (Carl Zeiss AG, Germany). The edge of the lesion was distinguished along the edema zone around the lesion, about $5 \mathrm{~mm}$ away, and the lesion was completely resected (Figs. $1 A$ and $B)$. The patient was given therapy against inflammation and for the prevention of epilepsy, rehydration and oral albendazole. The liver function of the patient was monitored regularly. 


\section{Results}

\section{Image analysis}

The results of brain MRI revealed a single lesion with multiple nodular and small cystic long $\mathrm{T} 1$ and short T2 signals in the left temporal lobe. On the contrast-enhanced scan, the lesion showed multiple ringshaped enhancements with an unclear boundary. The size of the lesion was $32 \mathrm{~mm} \times 18 \mathrm{~mm}$ (Figs. 2A to C).

The abdominal CT revealed masses of mixed density in the left lobe of the liver with a size of $138 \mathrm{~mm} \times$ $81 \mathrm{~mm}$, with a circular enhancement inside the lesion, irregular enlargement of the right lobe of the liver, and calcified masses. The left branch of the portal vein, hepatic vein and the intrahepatic segment of the inferior vena cava were not visible. The intrahepatic bile duct was dilated. According to WHO classification, it belonged to $P_{3} N_{1} M_{1}$. According to PIVM classification, it belonged to $P_{1-V, V I I I} l_{1} V_{1} M_{2} P_{1}$ (Figs. 3A and B).

\section{Serology}

The serum was strongly positive for antibodies against Echinococcus, and the eosinophil ratio was $20 \%$.

\section{Pathohistology and immunology}

The morphological results of HE staining were consistent with the characteristics of alveolar echinococcosis (Figs. 4A and B). The results of immunohistochemical staining showed positive staining for GFAP in peripheral glial cells and the Ki-67 index was less than $3 \%$ (Figs. 5A and B).

\section{Molecular genetic analysis}

After amplification of the cytochrome $\mathrm{C}$ oxidase gene by PCR using primers specific for Echinococcus, a fragment of the expected size of about $880 \mathrm{bp}$ was observed on the agarose gel after electrophoresis (Fig. 6). Sequence analysis identified the etiological agent from the lesion as the Asian strain of Echinococcus multilocularis with $100.00 \%$ sequence identity to the reference strains MH259774/69/68/64, KY446483/77/76/83/74, KY354088/84/83, KY328694/74 and KY205685 from China. The phylogenetic tree showed that the isolate was closely related to other Chinese isolates, clustered on one branch and far from other isolates (Figure 7).

\section{Differential diagnosis}

The results of lumbar puncture showed that the CSF was clear and transparent, the number of white cells was $1 \times 10^{6} / \mathrm{L}\left(1-10 \times 10^{6} / \mathrm{L}\right)$, and the CSF fluid pressure was normal. No abnormalities were found upon brain CTA and chest CT.

\section{Surgery and drug therapy}


Albendazole tablets were administered twice a day after admission, and the changes of liver function were monitored regularly. Microscopy revealed that the left temporal pole was very full, the sulcus gyri became shallow and flat, the cortex was beige, the boundary of the lesion was unclear, the lesion was firm, and there was no envelope. One piece of toughened grayish red and yellow tissue with a size of 40 $\mathrm{mm} \times 30 \mathrm{~mm}$ was excised. The cysts were solid and $0.2-0.4 \mathrm{~mm}$ in diameter. Gray inclusions were visible in the capsule (Figs. 8A and B).

\section{Outcome}

The patient's headache symptoms improved and no other neurological complications occurred. The brain MRI one month later after the discharge showed that the lesion had been fully resected (Figure 9).

\section{Discussion}

The Qinghai-Tibet Plateau, centered around Qinghai Province, is a high incidence area of AE. The patient described here is a herdsman with a history of close contact with cattle, sheep and dogs. The patient was diagnosed with liver AE in 2009 and received surgery. After the operation, the patient was required to take albendazole tablets orally. However, the patient had poor compliance, which is the most likely cause of the disease progression. He was diagnosed with echinococcosis of the right kidney in 2013 and underwent a right nephrectomy. The patient was asked to take albendazole orally again after the operation. After 2 months of intermittent administration, the patient stopped taking albendazole of his own accord. The patient lived in the Qinghai-Tibet Plateau, an economically underdeveloped area with inconvenient transportation and poor hygienic habits. Moreover, understanding of the disease is not high in said geographic area. Therefore, he did not cure the primary liver AE early and did not take albendazole orally as prescribed by the physician.

Many guidelines of the Expert Consensus in China ${ }^{[9,11-13]}$ and WHO ${ }^{[14]}$ recommend long-term oral albendazole for patients with echinococcosis. It is suggested that albendazole should be taken for life in patients with confirmed echinococcosis.

In this case, echinococcosis lesions invaded the left branch of the portal vein and the second hepatic portal, indicating that the disease should be classified as having reached $P_{3} N_{1} M_{1}$ and $P_{1-V, V I I I} I_{1} V_{1} M_{2} P_{1}$ stage. The diagnosis of intracranial metastasis was clear and indicated an advanced stage of disease ${ }^{[15]}$. According to the expert consensus on the diagnosis and treatment of hepatic hydatidosis type 2 (2015 edition ${ }^{[12]}$, patients with liver AE accompanied by intracranial metastasis are considered to have lost the opportunity for operation, and should be treated mainly with medication (albendazole). However, hepatectomy may also be considered for patients with complications of liver AE and stable intracranial lesions can be treated through medication. Therefore, the patient was treated with albendazole tablets on admission and the brain lesions were treated first. 
Primary liver alveolar echinococcosis usually results in multiple intracranial metastases ${ }^{[16]}$. In recent years, many cases of multiple brain $A E$ in different anatomical regions have been reported ${ }^{[17,18]}$. This case presented with a single brain metastasis, which is rare. Algros et al. ${ }^{[19]}$ showed that patients with AE should receive surgery as much as possible. The consensus of experts in China ${ }^{[9]}$ also indicates that the principal surgical treatment for brain AE should be selective excision of intracranial lesions, control of intracranial hypertension and relief of symptoms related to structural compression of nerves while antiechinococcal drugs should be used at the same time. Microsurgery induces less damage to brain tissue, and makes it easy to identify and completely resect lesions. It also reduces the possibility of anaphylactic shock due to leakage of cystic fluid and might cause less complications after operation, which could improve the therapeutic effect of surgery. The lesion was relatively superficial and located in a non-critical functional area. Therefore, we selected the microsurgical approach with resection along the edema zone around the lesion, and removed the lesion completely. The headache symptoms improved after the operation and no other neurological complications occurred. Therefore, this study suggested that if there is a single brain lesion in $\mathrm{AE}$, especially if it is located superficially and in a non-critical functional region, it should be removed as soon as possible. In the case of AE with multiple brain lesions, the safety and necessity of surgical treatment should be considered comprehensively according to the size, number, location of the lesions, as well as the amount of edema. The recurrence rates of brain echinococcosis after single surgery or chemotherapy were reported as 13 and $4 \%$, respectively ${ }^{[20]}$. Therefore, patients should be followed up regularly after discharge. When the intracranial condition is stable, the patients' liver condition should be further evaluated to determine whether the liver can receive surgical treatment. Bardonnet et al. ${ }^{[21]}$ reported the case of a 30-year-old AE patient with multiple metastases who received surgery and continued high-dose albendazole treatment, and achieved a good outcome. Our diagnostic and therapeutic program supports this study.

In addition, molecular genetic analysis of the lesion was carried out in this study and unequivocally identified the etiological agent as the Asian strain of Echinococcus multilocularis. In the era of increasing emphasis on precise medical care, this may provide a new approach for early disease screening of susceptible populations.

Although great progress has been made in basic research, clinical diagnosis and treatment of echinococcosis, AE is still a challenging disease with high morbidity, disability and mortality. Therefore, in areas with high incidence of the disease, it is very important to carry out extensive screening, and thereby enable early diagnosis and treatment of echinococcosis for the local residents.

\section{Conclusion}

Surgery is still the preferred treatment for AE patients with single or superficial lesions, especially in areas that are not functionally critical. Long-term albendazole treatment and regular follow-up are needed after operation. Early radical treatment and standardized drug treatment of primary liver AE are the key to 
avoid secondary brain echinococcosis. These aspects should be emphasized in the clinical practice to strengthen the comprehensive treatment of echinococcosis

\section{Abbreviations}

Alveolar echinococcosis

computed tomography

magnetic resonance imaging

enzyme linked immunosorbent assay

deoxyribonucleic acid

hematoxylin-eosin staining

glial fibrillary acidic protein

polymerase chain reaction

Molecular Evolutionary Genetics Analysis
$\mathrm{AE}$

CT

MRI

ELISA

DNA

HE

GFAP

PCR

\section{Declarations}

Ethics approval and consent to participate: This study was approved by the Ethics Committee of Qinghai Provincial People's Hospital.

Consent for publication: Informed consent was obtained from the patient.

Availability of data and materials: Not applicable.

Competing interests: The authors declare that they have no competing interests.

Funding: This study was supported by the National Natural Science Foundation of China (No. 81860370) and Qinghai Science and Technology Department Applied Basic Research Project (No.2015-ZJ-749).

Authors' contributions: Chaonan Du designed and writed articles. Qiangzhang, Mingfei Yang, Xiumin Han modified the article. Yanqiu Sun, Zhanying Ju, Xiaoxing Wei conducted the research and collected information. All authors read and approved the final manuscript.

Acknowledgements: The authors declare that they have no competing interests. 
Funding: This study was supported by the National Natural Science Foundation of China (No. 81860370) and Qinghai Science and Technology Department Applied Basic Research Project (No.2015-ZJ-749).

\section{References}

1. Deplazes P, Rinaldi L, Alvarez Rojas CA, et al. Global Distribution of Alveolar and Cystic Echinococcosis. Adv Parasitol 2017;95:315-493.

2.Torgerson PR, Keller K, Magnotta M, et al. The global burden of alveolar echinococcosis. PLoS Negl Trop Dis 2010, 4:e722.

3.Kern P, Bardonnet K, Renner E, et al. European echinococcosis registry: human alveolar echinococcosis, Europe, 1982-2000. Emerg Infect Dis 2003, 9: 343-349.

4.Altinors $\mathrm{N}$, Bavbek $\mathrm{M}$, Caner $\mathrm{HH}$, et al. Central nervous system hydatidosis in Turkey: a cooperative study and literature survey analysis of 458 cases. Neurosurg 2000, 93:1-8.

5.Debourgogne A, Goehringer F, Umhang G, et al. Primary cerebral alveolar echinococcosis: mycology to the rescue. J Clin Microbiol 2014, 52:692-694.

6.Tuzun Y, Solmaz I, Sengul G, et al. The complications of cerebral hydatid cyst surgery in children. Childs Nerv Syst 2010, 26:47-51.

7.Cai H, Guan Y, Ma X, et al. Epidemiology of Echinococcosis Among Schoolchildren in Golog Tibetan Autonomous Prefecture, Qinghai, China. Am J Trop Med Hyg 2017, 96:674-679.

8.Han XM, Cai QG, Wang W, et al. Childhood suffering: hyper endemic echinococcosis in Qinghai-Tibetan primary school students, China. Infect Dis Poverty 2018, 7:71.

9. Specialized Committee of Hydatidosis, Surgical Society of Chinese Medical Doctor Association. Expert consensus on the diagnosis and surgical treatment of central nervous system echinococcosis. Chin J Endemiol 2016, 35:625-628.

10.Kern P, Wen H, Sato N, et al. WHO classification of alveolar echinococcosis: principles and application. Parasitol Int 2006, 55:S283-287.

11.Kratzer W, Gruener B, Kaltenbach TE, et al. Proposal of an ultrasonographic classification for hepatic alveolar echinococcosis: Echinococcosis multilocularis Ulm classification-ultrasound. World J Gastroenterol 2015, 21:12392-12402.

12.Specialized Committee of Hydatidosis, Surgical Society of Chinese Medical Doctor Association. Expert consensus on the diagnosis and treatment of hepatic cystic and alveolar echinococcosis (2015 edition). Chinese Journal of Digestive Surgery 2015, 14:253-364. 
13. Li DS, Zhang LW, Zhang Z, et al. Expert consensus of standard diagnosis and treatment technology on pulmonary echinococcosis. Chin J Clin Torac Cardiovasc Surg 2015, 22: 799-802.

14.Guidelines for treatment of cystic and alveolar echinococcosis in humans. WHO Informal Working Group on Echinococcosis. Bull World Health Organ 1996, 74 :231-242区

15.Bresson-Hadni S, Delabrousse E, Blagosklonov O, et al. Imaging aspects and non-surgical interventional treatment in human alveolar echinococcosis. Parasitol Int 2006, 55:267-272.

16.Kantarci M, Bayraktutan U, Karabulut N, et al. Alveolar echinococcosis: spectrum of findings at crosssectional imaging. Radiographics 2012, 32: 2053-2070.

17. Tappe $D$, Weise $D$, Ziegler $U$, et al. Brain and lung metastasis of alveolar echinococcosis in a refugee from a hyperendemic area. J Med Microbiol 2008, 57:1420-1423.

18.Kantarci M, Ogul H, Bayraktutan U. Intracerebral alveolar echinococcosis. Headache 2012, 52:12961297.

19.Algros MP, Majo F, Bresson-Hadni S, et al. Intracerebral alveolar echinococcosis. Infection 2003, 31:6365.

20.Batçık OE, Öğrenci A, Koban O, Ekşi MŞ, Bilge T. Cerebral Alveolar Echinococcosis Concomitant with Liver and Lung Lesions in a Young Adult Patient: Case Report and Literature Review. Turkiye Parazitol Derg 2016, 40:169-171.

21.Bardonnet K, Vuitton DA, Grenouill F, et al. 30-yr course and favorable outcome of alveolar echinococcosis despite multiple metastatic organ involvement in a non-immune suppressed patient. Ann Clin Microbiol Antimicrob 2013, 12:1.

\section{Figures}



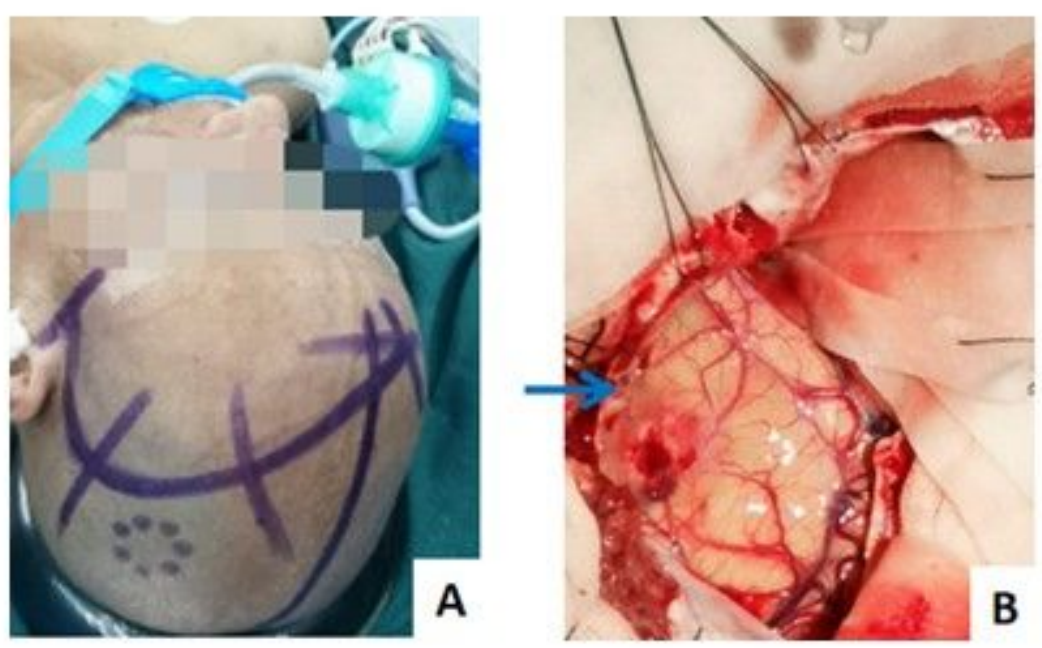

Figure 2

Surgical position and intraoperative view of the lesions A: The left pterional approach was selected, starting from $1 \mathrm{~cm}$ in front of the upper tragus of the left zygomatic arch, arching upward and ending at 2 $\mathrm{cm}$ in the middle line. B: Left temporal lesions were revealed. The left temporal pole was plump, the sulcus gyri became shallow and flat, and the cortex was beige.

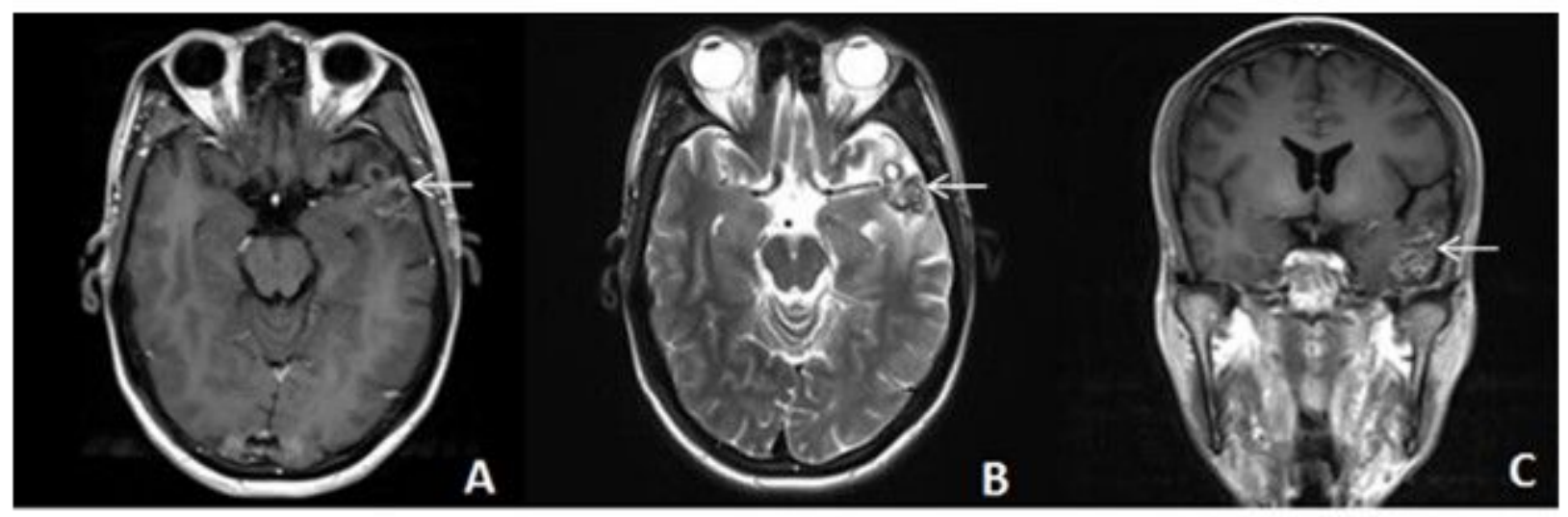

\section{Figure 4}

Brain MRI revealing a single lesion in the left temporal area A, B: multiple nodular and small cystic long T1 and short T2 signals in the left temporal lobe (white arrow). C: multiple ring-shaped enhancements with an unclear boundary (white arrow). 


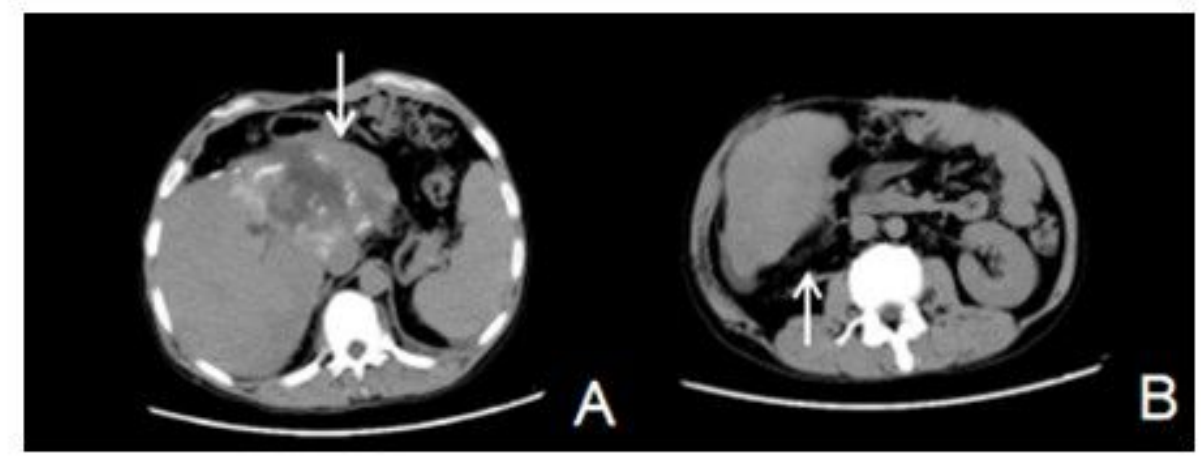

\section{Figure 6}

Abdominal CT A: Intrahepatic mixed density masses and masses with portal vein and second hepatic portal involvement. B: The right kidney is missing.
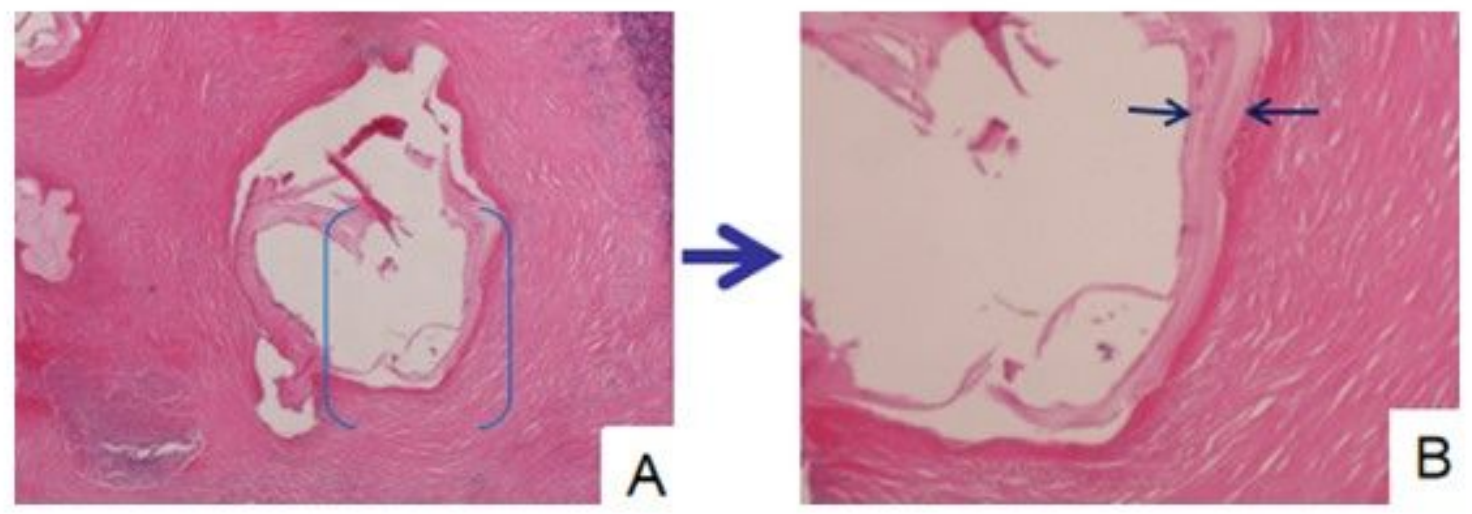

\section{Figure 8}

Representative micrograph of histological straining A: HE staining of lesion tissue at primary magnification, $2 \times 10$ times, B: Zoom of the selected cutout in image A (the original magnification was 10 $\times 10$ times). In the middle of the picture, there is a lamellar structure, which has no cellular components and is presumably a polysaccharide-protein complex. 

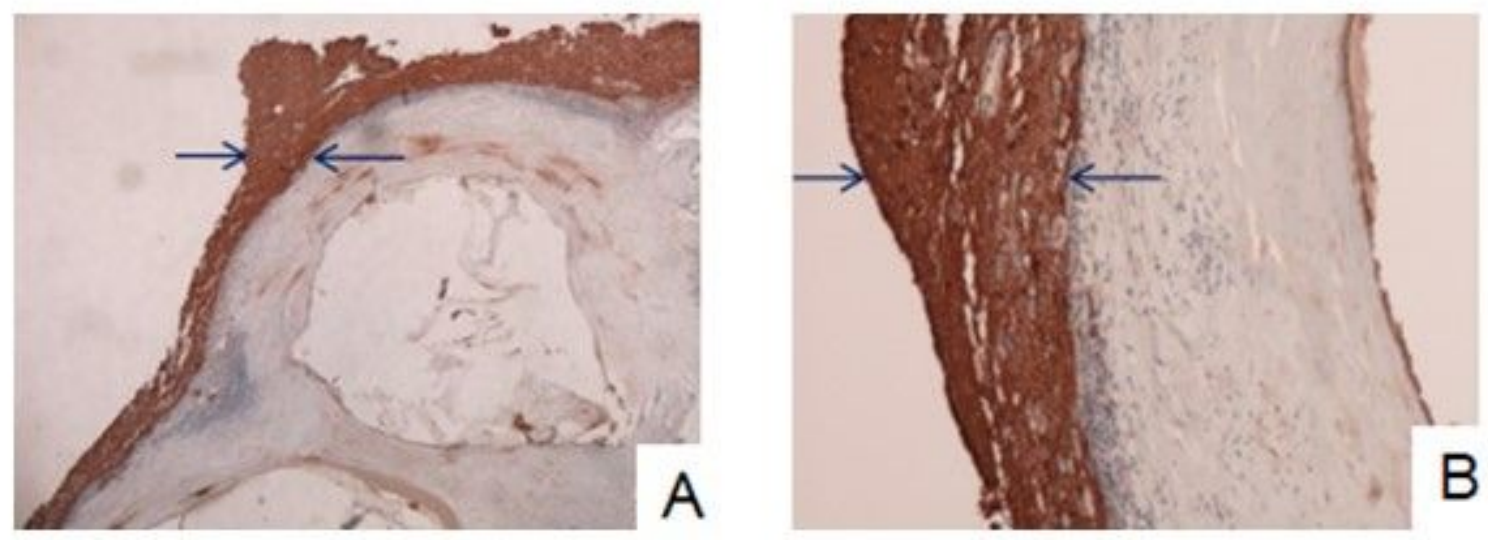

\section{Figure 10}

The results of immunohistochemical staining A, B: Immunohistochemical staining of glial cells around the lesion was positive for GFAP (blue arrow), confirming that the lesion was located in the intracranial region.

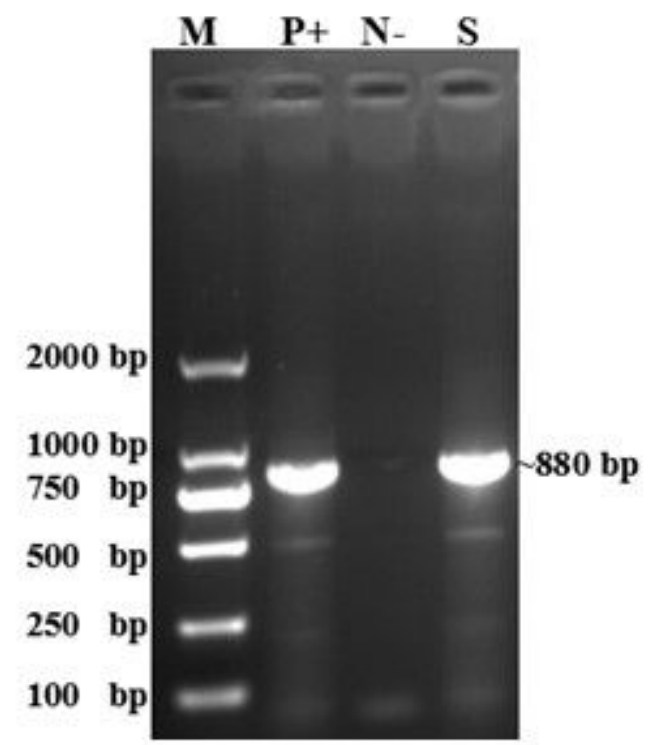

Figure 11 


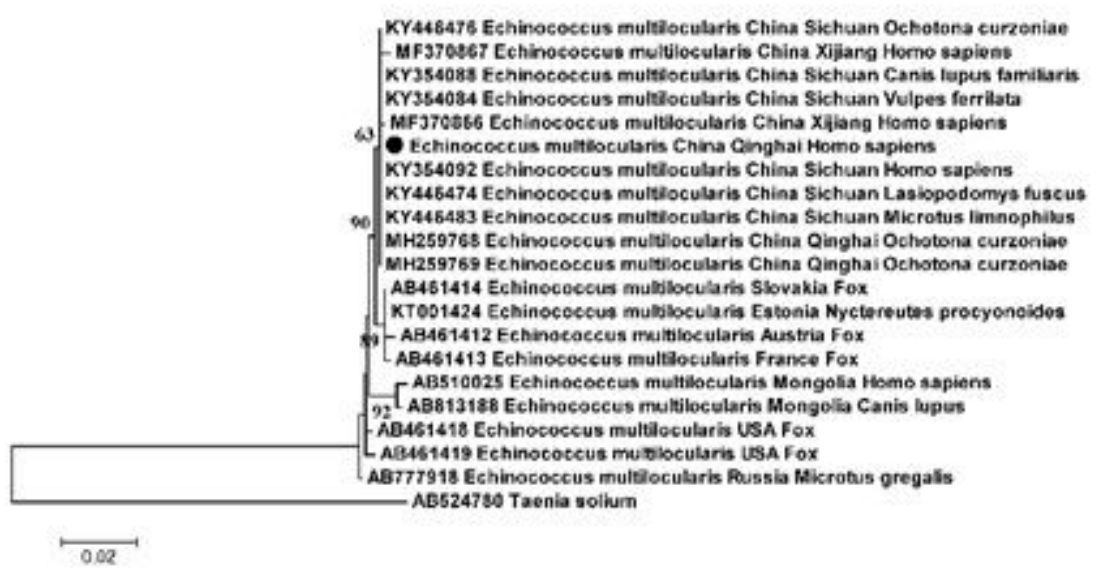

Figure 13

Evolutionary Tree Analysis of the COX1 Gene of different isolates of Echinococcus multilocularis.

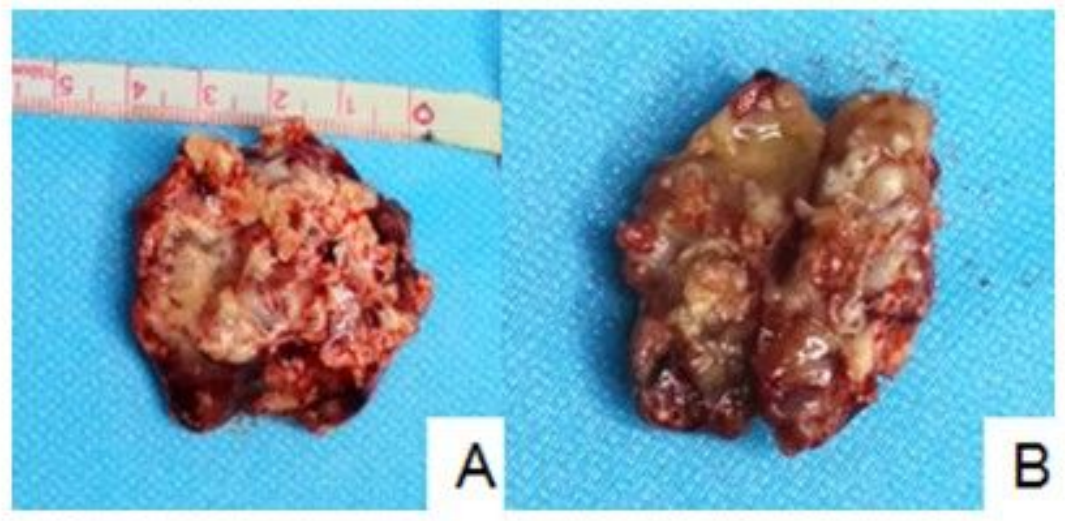


Figure 16

macroscopic appearance of the excised lesion A: Toughened grayish red and yellow tissue with a size of $40 \mathrm{~mm} \times 30 \mathrm{~mm}$ that was excised. B: The cysts were solid and 0.2-0.4mm in diameter. Gray inclusions were visible in the capsule

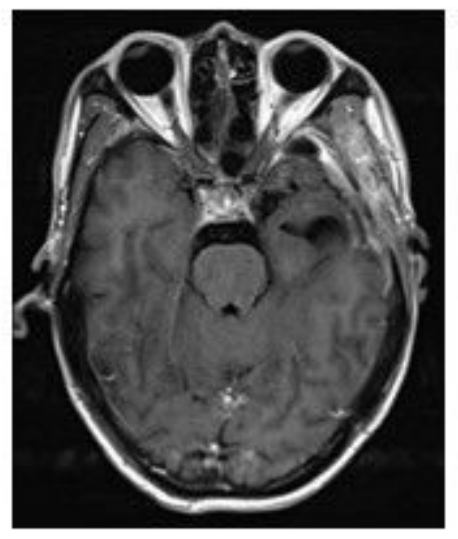

\section{Figure 18}

Brain MRI one month later after the discharge

\section{Supplementary Files}

This is a list of supplementary files associated with this preprint. Click to download.

- GraphicalAbstract.doc

- GraphicalAbstract.doc 